\title{
Mathematical Analysis of an Optimal Control Problem of Surface Water Pollution
}

\author{
Djibo Moustapha, Hamidou Haoua, Saley Bisso \\ Department of Mathematics and Informatics, Abdou Moumouni University, Niamey, Niger \\ Email:moustaphad530@gmail.com, hamidouhaoua@yahoo.com, bsaley@yahoo.fr
}

How to cite this paper: Moustapha, D., Haoua, H. and Bisso, S. (2017) Mathematical Analysis of an Optimal Control Problem of Surface Water Pollution. Applied Mathematics, 8, 164-179.

https://doi.org/10.4236/am.2017.82014

Received: January 10, 2017

Accepted: February 21, 2017

Published: February 24, 2017

Copyright $\odot 2017$ by authors and Scientific Research Publishing Inc. This work is licensed under the Creative Commons Attribution International License (CC BY 4.0).

http://creativecommons.org/licenses/by/4.0/

(c) (i) Open Access

\begin{abstract}
We present in this paper a new technique based on Gelfand's triplet [1] and include differential theory to make a theoretical analysis of an optimal control problem with constraints governed by coupled partial differential equations. This technique allowed us to give some theoretical results of existence and uniqueness of the solution of constraints and characterize the optimal control.
\end{abstract}

\section{Keywords}

Sand Dune Formation, Objective Function, Operators, State Equation

\section{Introduction}

The objective of this paper is to make a theoretical analysis of an optimal control problem with constraints governed by coupled partial differential equations.

The interest of this work is two-fold. Indeed firstly the resolution of optimal control problem with partial differential equations constraints is a challenge for the current research in the field both theoretically and numerically. Secondly, the mathematical model studied is of utmost importance in practice since the physical phenomenon is modeled through this paper concerning the pollution of surface water. The studied problem is very complex since the objective function is not explicitly defined in term of control directly. Hence there is a need to solve equations that govern constraints that are also complex as we couple the model which describes the pollutants dissolution with Navier-Stokes equations.

To overcome these difficulties, we built a new technique based on the Gelfand's triplet and theory of differential solutions to transform the partial differential equations into ordinary differential equations [1] [2].

The outline of this paper is as follows: in this second section we present the mathematical model; the third section is devoted to the main results of existence uniqueness and also estimation of the system; in the fourth section we conduct 
the characterization of the optimal control and we end this work with a conclusion and perspectives.

\section{Mathematical Model}

Let $\Omega$ be a bounded domain of $\mathbb{R}^{3}$, with boundary $\Gamma$ sufficiently regular. We denote by $\boldsymbol{x}=(x, y, z)$ an element of $\Omega$.

We consider the following model:

$$
\min J(v)=\frac{1}{2} \int_{0}^{T} \int_{\Omega}\left|D c(v)-z_{d}\right|^{2} \mathrm{~d} \boldsymbol{x} \mathrm{d} t+\frac{N}{2} \int_{0}^{T} \int_{\Omega}|v|^{2} \mathrm{~d} \boldsymbol{x} \mathrm{d} t,
$$

subject to

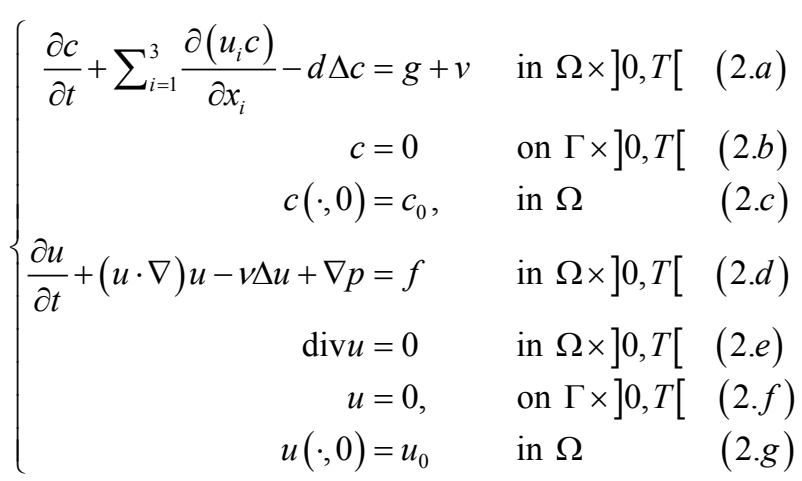

where:

- $z_{d}$ is an observation function; $D$ is a given linear continuous operator of observation; $N$ is a given positif real;

- $c$ denotes the concentration of pollutants, $g$ is a source term, $d$ is a diffusion coefficient, $v$ represented the control which allows to act on the system;

- $u=\left(u_{1}, u_{2}, u_{3}\right)$ and $p=p(\boldsymbol{x}, t)$ denote respectively the velocity and the pressure of water, $f$ external forces, $v$ the kinematic viscosity;

- Equations (2.a)-(2.c) modelling the transport and dissolution of pollutants;

- Systems (2.d)-(2.g) is Navier-Stokes equations.

We rewrite problems (1)-(2) in the following compact form:

$$
\left\{\begin{array}{l}
\text { Find } \quad u \in U_{a d} \quad \text { such that : } \\
J(u)=\min _{v \in U_{a d}} J(v)
\end{array}\right.
$$

where $U_{a d}$ denotes set of admissible controls. In this form it is easier to establish results of existence and uniqueness concerning the control when knowing some properties of $J$ and $U_{a d}$ [3] [4] [5].

\section{Main Results}

To make the theoretical study of system (2), we define the following Gelfand triplet:

$V \longmapsto H \longmapsto V^{*}$, where the injections are continuous, dense and compact.

We choose in this paper $V=H_{0}^{1}(\Omega), H=L^{2}(\Omega)$ and $V^{*}=\left(H_{0}^{1}(\Omega)\right)^{\prime}=H^{-1}(\Omega)$. 
We assume that there exist two positive constants $A$ and $A^{\star}$ such that the following inequality hold:

$$
\frac{1}{A^{*}}\|\cdot\|_{V^{*}} \leq\|\cdot\|_{H} \leq A\|\cdot\|_{V} .
$$

We also recall the following result concerning the Navier-Stokes equations whose proof can be found in [6].

Theorem 1. Let $u_{0}$ belonging to $L^{2}(\Omega)$ a divergence field. Then there exists a unique solution $u$ of the Navier-Stokes equations associated to the initial data $u_{0}$, such that

$$
u \in L^{\infty}\left([0, T] ; L^{2}(\Omega)\right) \cap L^{2}\left([0, T] ; H_{0}^{1}(\Omega)\right)
$$

and

$$
\forall t \geq 0, \quad \frac{1}{2}\|u(t)\|_{L^{2}(\Omega)}^{2}+\int_{0}^{T}\|\nabla u(\theta)\|_{L^{2}(\Omega)}^{2} \mathrm{~d} \theta \leq \frac{1}{2}\left\|u_{0}\right\|_{L^{2}(\Omega)}^{2}
$$

\subsection{Main Result 1}

This first result concerning existence and uniqueness of solution of the model that governs the dissolution of pollutants:

$$
\left\{\begin{aligned}
\frac{\partial c}{\partial t}+\sum_{i=1}^{3} \frac{\partial\left(u_{i} c\right)}{\partial x_{i}}-d \Delta c & =g+v & & \text { on } \Omega \times] 0, T[ \\
c & =0 & & \text { in } \Gamma \times] 0, T[ \\
c(\cdot, 0) & =0, & & \text { on } \Omega
\end{aligned}\right.
$$

For this we introduce Gelfand's triplet for the following operators:

$$
\Delta:=\left(\begin{array}{ccc}
-\frac{\partial^{2}}{\partial x^{2}} & 0 & 0 \\
0 & -\frac{\partial^{2}}{\partial y^{2}} & 0 \\
0 & 0 & -\frac{\partial^{2}}{\partial z^{2}}
\end{array}\right): V \rightarrow V^{*},
$$

and

$$
U \cdot \nabla:=\left(\begin{array}{ccc}
u_{1} \frac{\partial}{\partial x} & 0 & 0 \\
0 & u_{2} \frac{\partial}{\partial y} & 0 \\
0 & 0 & u_{3} \frac{\partial}{\partial z}
\end{array}\right): V \rightarrow H .
$$

We consider a function $C:[0, T] \rightarrow V$ defined by:

$$
C(t):=\left(\begin{array}{l}
k c(t, \cdot) \\
k c(t, \cdot) \\
k c(t, \cdot)
\end{array}\right) \text { where } k=\frac{1}{3}
$$

and 


$$
G=\left(\begin{array}{l}
k(g+v) \\
k(g+v) \\
k(g+v)
\end{array}\right)=G_{0}+V
$$

With these notations Equation (6) becomes:

$$
\left\{\begin{array}{l}
\dot{C}(t)+U \cdot \nabla C(t)+d \Delta C(t)=G(t) \quad \text { a.e } t \in[0, T] \\
C(0)=C_{0} \text { on } H,
\end{array}\right.
$$

where $\dot{C}$ denotes the derivative in the sense of the distributions of $C$ and $C_{0}=\left(\begin{array}{l}k c_{0} \\ k c_{0} \\ k c_{0}\end{array}\right)$.

We define the space of solutions by:

$$
W(0, T)=\left\{w \in L^{2}\left(0, T ; H_{0}^{1}(\Omega)\right), \dot{w} \in L^{2}\left(0, T ; H^{-1}(\Omega)\right)\right\},
$$

where $L^{2}\left(0, T ; H_{0}^{1}(\Omega)\right)$ and $L^{2}\left(0, T ; H^{-1}(\Omega)\right)$ are equipped with the respective norms:

$$
\|f\|_{L^{2}\left(0, T ; H_{0}^{1}(\Omega)\right)}=\left(\int_{0}^{T}\|f(t)\|_{H_{0}^{1}(\Omega)}^{2} \mathrm{~d} t\right)^{\frac{1}{2}}
$$

and

$$
\|f\|_{L^{2}\left(0, T ; H^{-1}(\Omega)\right)}=\left\{\int_{0}^{T}\left(\sup _{\phi \in H_{0}^{1}(\Omega)} \frac{(f(t), \phi(t))}{\|\phi\|_{H_{0}^{1}(\Omega)}}\right)^{2} \mathrm{~d} t\right\}^{\frac{1}{2}} \phi(t) \neq 0 .
$$

Theorem 2. If $U \in L^{\infty}\left(0, T ;\left[H_{0}^{1}(\Omega)\right]^{3}\right), \quad C_{0} \in H \quad$ and $\quad G \in L^{2}(0, T ; H)$ then there exists a unique state:

$$
C \in W(0, T) \cap L^{\infty}(0, T ; H),
$$

solution of the problem (11).

Proof. To proof uniqueness we first establish the following intermediate result:

$$
\begin{gathered}
\forall U \in L^{2}\left(0, T ;\left[H_{0}^{1}(\Omega)\right]^{3}\right) \text { and } C \in L^{2}(0, T ; V) \text { then for all } t \in[0, T]: \\
(U(t) \cdot \nabla C(t), C(t))_{V^{*}, V}=0 .
\end{gathered}
$$

Indeed,

$$
\begin{aligned}
& (U(t) \cdot \nabla C(t), C(t))_{V^{*}, V} \\
= & \int_{\Omega} U(t) \cdot \nabla C(t) C(t)=\int_{\Omega} \sum_{i=1}^{3} U_{i}(t) \frac{\partial C(t)}{\partial x_{i}} C(t) \\
= & \int_{\Gamma} \sum_{i=1}^{3} U_{i} C(t) C(t)-\int_{\Omega} \sum_{i=1}^{3}\left(U_{i}(t) \frac{\partial C(t)}{\partial x_{i}}+C(t) \frac{\partial U_{i}(t)}{\partial x_{i}}\right) C(t) \\
= & -\int_{\Omega} U(t) \cdot \nabla C(t) C(t)=-(U(t) \cdot \nabla C(t), C(t))_{V^{*}, V} \\
& 2(U(t) \cdot \nabla C(t) C(t))_{V^{*}, V}=0 \Rightarrow(U(t) \cdot \nabla C(t), C(t))_{V^{*}, V}=0 .
\end{aligned}
$$

Assuming existence of two solutions $C_{1}$ and $C_{2}$ of (11), let denote by 
$C=C_{1}-C_{2}$ then $C$ satisfies the following equation:

$$
\left\{\begin{array}{l}
\dot{C}(t)+d \Delta C_{1}(t)=U_{2} \cdot \nabla C_{2}(t)-U_{1} \cdot \nabla C_{1}(t) \text { a.e } t \in[0, T] \\
C(0)=0 \text { on } H .
\end{array}\right.
$$

by multiplying the first equation of (18) by $C$ and integrating over $[0, t]$, we obtain:

$$
\begin{aligned}
& \int_{0}^{t}(\dot{C}(\theta), C(\theta)) \mathrm{d} \theta+d \int_{0}^{t}(\Delta C(\theta), C(\theta)) \mathrm{d} \theta \\
&=\int_{0}^{t}\left(U_{2} \cdot \nabla C_{2}(t)-U_{1} \cdot \nabla C_{1}(t), C(\theta)\right) \mathrm{d} \theta \\
& \frac{1}{2}|C(t)|^{2}-\frac{1}{2}|C(0)|^{2}=-d \int_{0}^{t}\left(\Delta C_{1}(\theta)-\Delta C_{2}(\theta), C(\theta)\right) \mathrm{d} \theta \\
&-\int_{0}^{t}\left(U_{2} \cdot \nabla C_{2}(t), C_{2}(\theta)\right) \mathrm{d} \theta+\int_{0}^{t}\left(U_{1} \cdot \nabla C_{1}(t), C_{1}(\theta)\right) \mathrm{d} \theta
\end{aligned}
$$

using the monotony of $\Delta(t, \cdot)$, we deduce that, $\left(\Delta C_{1}(\theta)-\Delta C_{2}(\theta), C(\theta)\right) \geq 0$ and using relation (16) we deduce that:

$$
\begin{aligned}
& \frac{1}{2}|C(t)|^{2}-\frac{1}{2}|C(0)|^{2} \leq 0 \\
& |C(t)|^{2} \leq|C(0)|^{2},
\end{aligned}
$$

since $C(0)=0$, we have $|C(t)|=0 \Leftrightarrow C=0$ implying that $C 1=C 2$.

To prove existence of the solution we first prove that the problem approached of the problem model has a unique solution and that this approximate solution converges to the exact solution. Let consider the following approached problem $\left(P_{m}\right)$ of Equation (11) given by:

$$
\left\{\begin{array}{l}
\dot{C}_{m}(t)+U \cdot \nabla C_{m}(t)+\mathrm{d} \Delta C_{m}(t)=G_{m}(t) \quad \text { a.e } t \in[0, T] \\
C_{m}(0)=C_{0 m}
\end{array}\right.
$$

where $G_{m}(:)=\sum_{i=1}^{m}\left(G(\cdot), e_{j}\right) e_{j}$ and $C_{0 m}=\sum_{i=1}^{m}\left(C_{0}, e_{j}\right) e_{j}$.

We assume that this approximated problem is posed on the subspace $V_{m}=\operatorname{Vect}\left\{e_{1}, e_{2}, \cdots, e_{m}\right\}$ of the space $V$ which is separable, then there exists a sequence $\left(e_{m}\right)_{m \in \mathbb{N}^{*}}$ such that:

- $\forall m \in \mathbb{N}^{*}$, The first terms $e_{1}, e_{2}, \cdots, e_{n}$ are linearly independent;

- The set of linear combinations of elements of this sequence is dense in $V$ (and therefore in $H$ and $V^{*}$ ).

Proposition 3. Under the assumptions of theorem (2), $\forall m \geq 1$, (22) admits a unique solution $C_{m}$ which belongs to $V_{m}$.

Proof. The proof is based on the Carathéodory's theorem which we apply to the following function:

$$
\begin{aligned}
& F_{m}:[0, T] \times V_{m} \rightarrow \mathbb{R}^{m} \text { defined by } \\
& F_{m}(t, X)=\sum_{i=1}^{m}\left[\left(-\mathrm{d} \Delta C_{m}(t)-U \cdot \nabla X(t), e_{j}\right)+G_{m}(t)\right] .
\end{aligned}
$$

- $F_{m}$ is measurable as $\Delta(\cdot, v)$ and $U \cdot \nabla(\cdot, v)$ are measurable respectively in $V^{*}$ and $H$, this function is also continuous in $\mathrm{W}$ [7] [8];

- the condition of minoration on any compact of $V_{m}$ is assured by the fact 
that $\Delta(t, \cdot)$ is bounded a.e $t \in[0, T]$ in $V^{*}$ and $U \cdot \nabla(t, \cdot)$ is bounded in $H$ a.e $t \in[0, T]$.

Then, for every $X_{0} \in \Omega$, there exists at least one solution to the system:

$$
\left\{\begin{array}{l}
\dot{X}=F_{m}(t, X) \\
X(0)=X_{0}
\end{array}\right.
$$

which is therefore the solution of (22).

In order to demonstrate the convergence of the approximated solution to the exact solution, we establish the following priori estimation results.

Proposition 4. If $C$ is a solution of (11) then:

$$
\begin{aligned}
\|C\|_{L^{2}(0, T ; V)} \leq & \frac{1}{\sqrt{2 d}}\left|C_{0}\right|+\frac{1}{d}\|G\|_{L^{2}\left(0, T ; V^{*}\right)} \\
\|C\|_{L^{\infty}(0, T ; H)} \leq & \left|C_{0}\right|+\sqrt{\frac{2}{d}}\|G\|_{L^{2}\left(0, T ; V^{*}\right)} \\
\|\dot{C}\|_{L^{2}\left(0, T ; V^{*}\right)} \leq & {\left[\sqrt{\frac{d}{2}}\left|C_{0}\right|+\|G\|_{L^{2}\left(0, T ; V^{*}\right)}\right]\left[A+\sqrt{\frac{2}{d}}\|U\|_{L^{2}\left(0, T ;\left[H_{0}^{1}(\Omega)\right]^{3}\right)}\right] } \\
& +\|G\|_{L^{2}\left(0, T ; V^{*}\right)}
\end{aligned}
$$

Proof. To prove relation (25) we form the scalar product of the first equation of Equation (11) with $C$ and integrate over $[0, T]$, we obtain:

$$
\begin{aligned}
& (\dot{C}(t), C(t))_{V^{*}, V}+(U \cdot \nabla C(t), C(t))_{V^{*}, V}+d(\Delta C(t), C(t))_{V^{*}, V} \\
& =(G(t), C(t))_{V^{*}, V} \\
& (\dot{C}(t), C(t))_{V^{*}, V}+d(\Delta C(t), C(t))_{V^{*}, V}=(G(t), C(t))_{V^{*}, V} .
\end{aligned}
$$

Integrate over $[0, T]$

$$
\int_{0}^{T}(\dot{C}(t), C(t))_{V^{*}, V} \mathrm{~d} t+d \int_{0}^{T}(\Delta C(t), C(t))_{V^{*}, V} \mathrm{~d} t=\int_{0}^{T}(G(t), C(t))_{V^{*}, V} \mathrm{~d} t .
$$

then

$$
\begin{aligned}
\int_{0}^{T}(\dot{C}(t), C(t))_{V^{*}, V} \mathrm{~d} t= & (C(T), C(T))_{L^{2}(\Omega)}-(C(0), C(0))_{L^{2}(\Omega)} \\
& -\int_{0}^{T}(\dot{C}(t), C(t))_{V^{*}, V} \mathrm{~d} t \\
\int_{0}^{T}(\dot{C}(t), C(t))_{V^{*}, V} \mathrm{~d} t= & \frac{1}{2}|C(T)|^{2}-\frac{1}{2}|C(0)|^{2} .
\end{aligned}
$$

We have: $\|\Delta C\|_{V^{*}} \leq \alpha_{1}(t)+\alpha_{2}(t)\|C(t)\|_{V} \leq\|C(t)\|_{V}$ :

$$
\int_{0}^{T}(\Delta C(t), C(t))_{V^{*}, V} \mathrm{~d} t \leq \int_{0}^{T}\|\Delta C\|_{V^{*}}^{2} \mathrm{~d} t \leq \int_{0}^{T}\|C(t)\|_{V}^{2} \mathrm{~d} t=\|C\|_{L^{2}(0, T ; V)}^{2}
$$

and we deduce the following successive inequalities

$$
\begin{aligned}
& \int_{0}^{T}(G(t)+V(t), C(t))_{V^{*}, V} \mathrm{~d} t \leq \int_{0}^{T}\|G(t)\|_{V^{*}}\|C(t)\|_{V} \mathrm{~d} t \\
& \leq\|G\|_{L^{2}\left(0, T ; V^{*}\right)}\|C\|_{L^{2}(0, T ; V)} . \\
& \frac{1}{2}|C(T)|^{2}-\frac{1}{2}|C(0)|^{2}+d\|C\|_{L^{2}(0, T ; V)}^{2} \leq\|G\|_{L^{2}\left(0, T ; V^{*}\right)}\|C\|_{L^{2}(0, T ; V)} .
\end{aligned}
$$




$$
\begin{aligned}
& d\|C\|_{L^{2}(0, T ; V)}^{2}-\|G\|_{L^{2}\left(0, T ; V^{*}\right)}\|C\|_{L^{2}(0, T ; V)} \leq \frac{1}{2}|C(0)|^{2}-\frac{1}{2}|C(T)|^{2} \\
& \leq \frac{1}{2}|C(0)|^{2} \\
& {\left[\sqrt{d}\|C\|_{L^{2}(0, T ; V)}-\frac{1}{2 \sqrt{d}}\|G\|_{L^{2}\left(0, T ; V^{*}\right)}\right]^{2} } \leq \frac{1}{2}|C(0)|^{2}+\frac{1}{4 d}\|G\|_{L^{2}\left(0, T ; V^{*}\right)} \\
& \leq\left[\frac{1}{\sqrt{2}}|C(0)|+\frac{1}{2 \sqrt{d}}\|G\|_{L^{2}\left(0, T ; V^{*}\right)}\right]^{2} \\
& \sqrt{d}\|C\|_{L^{2}(0, T ; V)}-\frac{1}{2 \sqrt{d}}\|G+V\|_{L^{2}\left(0, T ; V^{*}\right)} \leq\left[\frac{1}{\sqrt{2}}|C(0)|+\frac{1}{2 \sqrt{d}}\|G\|_{L^{2}\left(0, T ; V^{*}\right)}\right] \\
& \sqrt{d}\|C\|_{L^{2}(0, T ; V)} \leq\left[\frac{1}{\sqrt{2}}|C(0)|_{\left.+\frac{1}{\sqrt{d}}\|G\|_{L^{2}\left(0, T ; V^{*}\right)}\right]}\right. \\
&\|C\|_{L^{2}(0, T ; V)} \leq \frac{1}{\sqrt{2 d}}\left|C_{0}\right|+\frac{1}{d}\|G\|_{L^{2}\left(0, T ; V^{*}\right)} .
\end{aligned}
$$

To establish relation (26) we integrate the scalar product over $[0, t] \subset[0, T]$ :

$$
\begin{aligned}
& \int_{0}^{t}(\dot{C}(s), C(s))_{V^{*}, V} \mathrm{~d} s+d \int_{0}^{t}(\Delta C(s), C(s))_{V^{*}, V} \mathrm{~d} s \\
& =\int_{0}^{t}(G(s), C(s))_{V^{*}, V} \mathrm{~d} s . \\
& \frac{1}{2}|C(t)|^{2}-\frac{1}{2}|C(0)|^{2}+d\|C\|_{L^{2}(0, T ; V)}^{2} \leq\|G\|_{L^{2}\left(0, T ; V^{*}\right)}\|C\|_{L^{2}(0, T ; V)} . \\
& \frac{1}{2}|C(t)|^{2} \leq \frac{1}{2}|C(0)|^{2}-d\|C\|_{L^{2}(0, T ; V)}^{2}+\|G\|_{L^{2}\left(0, T ; V^{*}\right)}\|C\|_{L^{2}(0, T ; V)} \\
& \quad \leq \frac{1}{2}|C(0)|^{2}+\|G\|_{L^{2}\left(0, T ; V^{*}\right)}\|C\|_{L^{2}(0, T ; V)},
\end{aligned}
$$

Using (25) we have:

$$
\begin{aligned}
\frac{1}{2}|C(t)|^{2} & \leq \frac{1}{2}|C(0)|^{2}+\|G+V\|_{L^{2}\left(0, T ; V^{*}\right)}\left[\frac{1}{\sqrt{2 d}}\left|C_{0}\right|+\frac{1}{d}\|G\|_{L^{2}\left(0, T ; V^{*}\right)}\right] \\
& \leq \frac{1}{2}|C(0)|^{2}+\frac{1}{\sqrt{2 d}}\left|C_{0}\right|\|G\|_{L^{2}\left(0, T ; V^{*}\right)}+\frac{1}{d}\|G\|_{L^{2}\left(0, T ; V^{*}\right)}^{2} \\
|C(t)|^{2} & \leq|C(0)|^{2}+\sqrt{\frac{2}{d}}\left|C_{0}\right|\|G\|_{L^{2}\left(0, T ; V^{*}\right)}\left[\sqrt{\frac{2}{d}}\|G\|_{L^{2}\left(0, T ; V^{*}\right)}\right]^{2} \\
& \left.\leq|| C(0) \mid+\sqrt{\frac{2}{d}}\|G\|_{L^{2}\left(0, T ; V^{*}\right)}\right]^{2} \\
|C(t)|^{2} & \leq|C(0)|+\sqrt{\frac{2}{d}}\|G\|_{L^{2}\left(0, T ; V^{*}\right)},
\end{aligned}
$$

then we have

$$
\|C\|_{L^{\infty}(0, T ; H)} \leq\left|C_{0}\right|+\sqrt{\frac{2}{d}}\|G\|_{L^{2}\left(0, T ; V^{*}\right)}
$$

To proof relation (27) we use the fact that the Laplacian is a bounded linear operator and the following lemma: 
Lemma 3.1. $\forall w \in\left[H_{0}^{1}(\Omega)\right]^{3}$ and for all $z \in H_{0}^{1}(\Omega)$ there exist two constants $\beta$ and $\gamma$ such as:

$$
|w \cdot \nabla z| \leq \gamma \beta\|w\|_{\left[H_{0}^{1}(\Omega)\right]^{3}}\|z\|_{H_{0}^{1}(\Omega)} .
$$

Proof. For all $w \in\left[H_{0}^{1}(\Omega)\right]^{3}$ and for all $z \in H_{0}^{1}(\Omega)$, we have:

$$
\left.|w \cdot \nabla z|^{2}=\int_{\Omega}\left|\sum_{i=1}^{3} w_{i} \frac{\partial z}{\partial x_{i}}\right|^{2} \mathrm{~d} x \leq \int_{\Omega}\left|\sum_{i=1}^{3} w_{i}\right|^{2}\left|\sum_{i=1}^{3} \frac{\partial z}{\partial x_{i}}\right|^{2} \leq\|w\|_{\left[L^{2}(\Omega)\right.}^{2}\right]\|z\|_{H^{1}(\Omega)}^{2} .
$$

According to Poincaré inequality there exist $\gamma>0$ such as $\|w\|_{\left[L^{2}(\Omega)\right]^{3}} \leq \gamma\|\nabla w\|_{\left[L^{2}(\Omega)\right]^{3}}$ :

$$
\begin{aligned}
& \left.\left.|w \cdot \nabla z|^{2} \leq \gamma^{2}|\nabla w|_{\left[L^{2}(\Omega)\right.}^{2}\right]\right]^{3}\|z\|_{H^{1}(\Omega)}^{2} \\
& |w \cdot \nabla z|^{2} \leq \gamma^{2}\left\|\left.w\right|_{\left[H_{0}^{1}(\Omega)\right]^{2}} ^{2}\right\| z \|_{H^{1}(\Omega)}^{2}
\end{aligned}
$$

Norms \|\|$_{H_{0}^{1}}$ and $\|\cdot\|_{H^{1}}$ are equivalent in $H^{1}(\Omega)$, so that there exist a positive constant $\beta$ such as:

$$
\left.|w \cdot \nabla z|^{2} \leq \gamma^{2} \beta^{2} \|\left. w\right|_{\left[H_{0}^{1}(\Omega)\right.} ^{2}\right]^{3}\|z\|_{H_{0}^{1}(\Omega)}^{2},
$$

then we have

$$
|w \cdot \nabla z| \leq \gamma \beta\|w\|_{\left[H_{0}^{1}(\Omega)\right]^{3}}\|z\|_{H^{1}(\Omega)}
$$

Lemma 3.2. If $U \in L^{2}\left(0, T ;\left[H_{0}^{1}(\Omega)\right]^{3}\right)$ and $C \in L^{2}(0, T ; V)$ then $U \cdot \nabla C \in L^{2}(0, T ; H)$. Furthermore

$$
\|U \cdot \nabla C\|_{L^{2}(0, T ; H)} \leq \gamma \beta\|U\|_{L^{2}\left(0, T ;\left[H_{0}^{1}(\Omega)\right]^{3}\right)}\|C\|_{L^{2}(0, T ; V)}
$$

Proof. For all $U \in L^{2}\left(0, T ;\left[H_{0}^{1}(\Omega)\right]^{3}\right)$ and for all $C \in L^{2}(0, T ; V)$, we have:

$$
\|U \cdot \nabla C\|_{L^{2}(0, T ; H)}^{2}=\int_{0}^{T}|U \cdot \nabla C|^{2} \mathrm{~d} t .
$$

According to Lemma (3.1) we have:

$$
\begin{aligned}
& \|U \cdot \nabla C\|_{L^{2}(0, T ; H)}^{2} \leq \gamma^{2} \beta^{2} \int_{0}^{T}\|U(t)\|_{\left[H_{0}^{1}(\Omega)\right]^{3}}^{2} \mathrm{~d} t \int_{0}^{T}\|C(t)\|_{H_{0}^{1}(\Omega)}^{2} \mathrm{~d} t, \\
& \|U \cdot \nabla C\|_{L^{2}(0, T ; H)} \leq \gamma \beta\|U\|_{\left[H_{0}^{1}(\Omega)\right]^{3}}^{2} \mathrm{~d} t\|C\|_{H_{0}^{1}(\Omega)},
\end{aligned}
$$

Consider $\phi \in L^{2}(0, T ; V)$ and we form the scalar product of the first equation of (11) with $\phi$ :

$$
\begin{aligned}
& \begin{aligned}
&(\dot{C}(t), \phi(t))_{V^{*}, V}+(U \cdot \nabla C(t), \phi(t))_{V^{*}, V}+d(\Delta C(t), \phi(t))_{V^{*}, V} \\
&=(G(t), \phi(t))_{V^{*}, V} \\
& \begin{aligned}
(\dot{C}(t), \phi(t))_{V^{*}, V}= & -(U \cdot \nabla C(t), \phi(t))_{V^{*}, V}-d(\Delta C(t), \phi(t))_{V^{*}, V} \\
& +(G(t), \phi(t))_{V^{*}, V}
\end{aligned} \\
&\left|\int_{0}^{T}(\dot{C}(t), \phi(t))_{V^{*}, V}\right| \mathrm{d} t \leq d\left|\int_{0}^{T}(\Delta C(t), \phi(t))_{V^{*}, V} \mathrm{~d} t\right|+\left|\int_{0}^{T}(U \cdot \nabla C(t), \phi(t))_{V^{*}, V} \mathrm{~d} t\right| \\
& \quad+\left|\int_{0}^{T}(G(t), \phi(t))_{V^{*}, V}\right| \mathrm{d} t
\end{aligned}
\end{aligned}
$$




$$
\begin{aligned}
& \left|\int_{0}^{T}(\Delta C(t), \phi(t))_{V^{*}, V}\right| \mathrm{d} t \leq \int_{0}^{T}|\Delta C(t)|\|\phi(t)\| \leq\|\Delta C\|_{V^{*}} \leq A\|C\|_{L^{2}(0, T ; V)} \\
& \left|\int_{0}^{T}(U \cdot \nabla C(t), \phi(t))_{V^{*}, V} \mathrm{~d} t\right| \leq \gamma \beta\|U\|_{L^{2}\left(0, T ;\left[H_{0}^{1}(\Omega)\right]^{3}\right)}\|C\|_{L^{2}(0, T ; V)} \cdot \\
& \left|\int_{0}^{T}(U \cdot \nabla C(t), \phi(t))_{V^{*}, V} \mathrm{~d} t\right| \leq\|U\|_{L^{2}\left(0, T ;\left[H_{0}^{1}(\Omega)\right]^{3}\right)}\|C\|_{L^{\infty}(0, T ; H)} .
\end{aligned}
$$

Finally we get

$$
\begin{aligned}
& \left|\int_{0}^{T}(\dot{C}(t), \varphi(t))_{V^{*}, V}\right| \mathrm{d} t \\
& \leq\left[d A\|C\|_{L^{2}(0, T ; V)}+\|U\|_{L^{2}\left(0, T ;\left[H_{0}^{1}(\Omega)\right]^{3}\right)}\|C\|_{L^{\infty}(0, T ; H)}+\|G\|_{L^{2}\left(0, T ; V^{*}\right)}\right],
\end{aligned}
$$

Using estimates (25) and (26), we obtain:

$$
\begin{aligned}
\|\dot{C}\|_{L^{2}\left(0, T ; V^{*}\right)} \leq & \frac{A d}{\sqrt{2 d}}|C(0)|+\frac{A d}{d}\|G\|_{L^{2}\left(0, T ; V^{*}\right)}+\|U\|_{L^{2}\left(0, T ;\left[H_{0}^{1}(\Omega)\right]^{3}\right)} \\
& +\sqrt{\frac{2}{d}}\|U\|_{L^{2}\left(0, T ;\left[H_{0}^{1}(\Omega)\right]^{3}\right)}\|G\|_{L^{2}\left(0, T ; V^{*}\right)}+\|G\|_{L^{2}\left(0, T ; V^{*}\right)},
\end{aligned}
$$

To achieve the proof of Theorem 2 it must be shown that the approximate solution $\left(C_{m}\right)$ converges to the exact solution in $W(0, T)$. By construction, the result of initial states $\left(C_{0 m}\right)_{m \in \mathbb{N}^{*}}$ converges to $C_{0}$ in $H$.

Using the relation (25), we deduce that the terms of the sequence $\left(C_{m}\right)$ is bounded in $L^{2}(0, T ; V)$ which is a reflexive Banach space. We can extract a subsequence that is weakly convergent in $\sigma\left(L^{2}(0, T ; V), L^{2}\left(0, T ; V^{*}\right)\right)$.

Let denote by $C_{\phi(n)}$ this subsequence where $\phi: \mathbb{N} \rightarrow \mathbb{N}$ is a function strictly increasing such that:

$$
C_{\phi(n)} \rightarrow C_{1} \text { weakly in } L^{2}(0, T ; V)
$$

From relation (26) we deduce that the sequence $\left(C_{m}\right)$ are bounded in $L^{\infty}(0, T ; H)$ which is the dual of $L^{1}(0, T ; H)$. Then there exists a sub-sequence which converges weakly towards $C_{2}$ such as:

$$
C_{\phi(n)} \rightarrow C_{2} \text { weakly in } L^{\infty}(0, T ; V) ;
$$

Using relation (27) we obtain the weak convergence $\dot{C}_{\phi(n)}$ to $\alpha \in L^{2}\left(0, T ; V^{*}\right)$ i.e:

$$
\dot{C}_{\phi(n)} \rightarrow \alpha \text { weakly in } L^{2}\left(0, T ; V^{*}\right)
$$

According to [9] [10], we deduce that $C=C_{1}=C_{2}$ and $\dot{C}=\alpha$.

On the other hand let $j \in \mathbb{N}$ fixed, $\forall m \in \mathbb{N}$ denoting by $\left(C_{m}\right)$ the unique solution of $\left(P_{m}\right)$ then when $m \rightarrow+\infty$ we obtain:

$$
\left(U \cdot \nabla C_{m}(t)-d \Delta C_{m}(t)-G_{m}(t), e_{j}\right) \rightarrow\left(U \cdot \nabla C(t)-d \Delta C(t)-G(t), e_{j}\right)
$$

and

$$
\left(\dot{C}_{m}(t), e_{j}\right) \rightarrow\left(\dot{C}(t), e_{j}\right) .
$$

Since the family $\left\{e_{j}, j \in \mathbb{N}^{*}\right\}$ is dense in $V$ thus: 


$$
\dot{C}(t)+U \cdot \nabla C(t)+d \Delta C(t)=G(t) p \cdot p t \in[0, T] .
$$

Also to [10], we can extract a subsequence noted again $C_{n}$ such as $p . p t \in[0, T]$, $\left(C_{n}(t)\right)_{\mathbb{N}^{*}}$ converges to $C(t)$. Considering the continuous representative $C$, we obtain strong convergence $\left(C_{n}(0)\right)_{\mathbb{N}^{*}}$ towards $C(0)$.

\subsection{Regularity of the Solution}

Let $\bar{C}$ be the unique solution of (11) and $\bar{C}+q$ a perturbed one of (11). We suppose that $C_{0}$ and $\lambda \in H, F, G \in L^{2}\left(0, T, V^{*}\right)$ and $U_{a}$, $U_{b} \in L^{\infty}\left(0, T ;\left[H_{0}^{1}(\Omega)\right]^{3}\right)$, then $q$ satisfies the following equation:

$$
\left\{\begin{array}{l}
\dot{q}(t)+U_{a} \cdot \nabla q(t)+U_{b} \cdot \nabla(\bar{C}(t)+q(t))+d \Delta q(t)=F(t) \quad \text { a.e } t \in[0, T] \\
q(0)=\lambda
\end{array}\right.
$$

Effect the scalar product of (40) by $q(t)$ and integrate over $[0, t] \subset[0, T]$, we obtain:

$$
\begin{aligned}
& \frac{1}{2}|q(t)|^{2}-\frac{1}{2}|\lambda|^{2}+\int_{0}^{t}\left(U_{a} \cdot \nabla q(s), q(s)\right) \mathrm{d} s+\int_{0}^{t}\left(U_{b} \cdot \nabla(\bar{C}(s)+q(s)), q(s)\right) \mathrm{d} s \\
& +d \int_{0}^{t}(\Delta q(t), q(t))=\int_{0}^{t}(F(s), q(s)) \mathrm{d} s .
\end{aligned}
$$

$\Delta$ being monotonous then:

$$
\frac{1}{2}|q(t)|^{2}-\frac{1}{2}|\lambda|^{2}+\int_{0}^{t}\left(U_{b} \cdot \nabla(\bar{C}(t)+q(t), q(t))\right) \mathrm{d} s=\int_{0}^{t}(F(t), q(t)) \mathrm{d} s .
$$

By Lemma 3.2, we have:

$$
\begin{aligned}
|q(t)|^{2} \leq & |\lambda|^{2}+2 \beta \gamma \int_{0}^{t}\left\|U_{b}\right\|_{\left[H_{0}^{1}(\Omega)\right]^{3}}\|\bar{C}(s)+q(s)\|_{H_{0}^{1}(\Omega)}\|q(s)\|_{H_{0}^{1}(\Omega)} \mathrm{d} s \\
& +2 \int_{0}^{t}\|F(s)\|_{H^{-1}(\Omega)}\|q(s)\|_{H_{0}^{1}(\Omega)}
\end{aligned}
$$

Let:

$$
\eta(s)=2 \beta \gamma\left\|U_{b}\right\|_{\left[H_{0}^{1}(\Omega)\right]^{3}}\|\bar{C}(s)+q(s)\|_{H_{0}^{1}(\Omega)}+\|F(s)\|_{H^{-1}(\Omega)}
$$

then

$$
|q(t)|^{2} \leq|\lambda|^{2}+\int_{0}^{t} \eta(s)\|q(s)\|_{H_{0}^{1}(\Omega)} .
$$

According to Willet-Wong's inequality we deduce:

$$
|q(t)| \leq|\lambda|+\frac{1}{2} \int_{0}^{t} \eta(s) \mathrm{d} s,
$$

Using relation (25), one can have a priori estimation of $q$ solution of (40). There exist a constant $M_{\lambda, W, F}$ as if $|\lambda|,\|F\|_{L^{2}\left(0, T, H^{-1}(\Omega)\right)}$ are increased then:

$$
\begin{aligned}
& |q(t)| \leq|\lambda|+\beta \gamma \int_{0}^{t} M_{\lambda, W, H}\left\|U_{b}\right\|_{\left[H_{0}^{1}(\Omega)\right]^{3}} \mathrm{~d} s+\int_{0}^{t}\|F(s)\|_{H^{-1}(\Omega)} \mathrm{d} s \\
& |q(t)| \leq|\lambda|+\beta \gamma M_{\lambda, W, H}\left\|U_{b}\right\|_{L^{\infty}}\left(0, T ;\left[H_{0}^{1}(\Omega)\right]^{3}\right)+\|F(s)\|_{L^{2}\left(0, T ; H^{-1}(\Omega)\right)} .
\end{aligned}
$$

Let $\rho=\max \left\{1, \beta \gamma M_{\lambda, W, H}\right\}$, then:

$$
|q(t)| \leq \rho|\lambda|+\left\|U_{b}\right\|_{L^{\infty}\left(0, T ;\left[H_{0}^{1}(\Omega)\right]^{3}\right)}+\|F(s)\|_{L^{2}\left(0, T ; H^{-1}(\Omega)\right)}
$$




\subsection{Main Result 2}

The space of admissible controls considered in this paper is $U_{a d}=L^{2}(0, T ; H)$.

Theorem 5 The optimal control problem (3) has a unique solution $W \in U_{a d}$ And we have the following inequality: $\forall V \in U_{a d}$ :

$$
\|W-V\|_{L^{2}(0, T ; H)} \leq \frac{4}{N}[J(V)-J(W)] .
$$

Proof. to proof this result, one can proof that the functional $J$ is inferior semicontinuous, strongly convex and differentiable.

Let us proof that $J$ is inferior semi-continuous. We consider a sequence $\left(C_{n}, V_{n}\right)$ in $L^{2}\left(0, T ; H_{0}^{1}(\Omega)\right) \times L^{2}(0, T ; H)$ which converges strongly to $(C, V)$, we can extract a subsequence also denoted by $\left(C_{n}, V_{n}\right)$ such that:

$$
\left(C_{n}(t), V_{n}(t)\right) \rightarrow(C(t), V(t)) \text { a.e in } H_{0}^{1}(\Omega) \times L^{2}(\Omega) .
$$

Let denote by $L(C(t), V(t))=X(C(t))+Y(V(t))$ with:

$$
\begin{aligned}
& X: H_{0}^{1}(\Omega) \rightarrow\left[0,+\infty\left[\quad C(t) \rightarrow X(C(t))=\int_{\Omega}\left|D C(v)-z_{d}\right|^{2} \mathrm{~d} \boldsymbol{x}\right.\right. \\
& Y: L^{2}(\Omega) \rightarrow\left[0,+\infty\left[\quad V(t) \rightarrow Y(V(t))=\int_{\Omega}|V|^{2} \mathrm{~d} \boldsymbol{x}\right.\right.
\end{aligned}
$$

$X$ and $Y$ are inferior semi-continuous then:

$$
L(C(t), V(t)) \leq \liminf _{n \rightarrow \infty} L\left(C_{n}(t), V_{n}(t)\right)
$$

Apply Fatou's lemma to the sequence $L\left(C_{n}(t), V_{n}(t)\right)$ we obtain:

$$
\int_{0}^{T} \liminf _{n \rightarrow \infty} L\left(C_{n}(t), V_{n}(t)\right) \mathrm{d} t \leq \liminf _{n \rightarrow \infty} \int_{0}^{T} L\left(C_{n}(t), V_{n}(t)\right) \mathrm{d} t,
$$

according to (45) we have:

$$
\begin{aligned}
\frac{1}{2} \int_{0}^{T} \int_{\Omega}\left|D C(v)-z_{d}\right|^{2} \mathrm{~d} \boldsymbol{x} \mathrm{d} t+\frac{N}{2} \int_{0}^{T} \int_{\Omega}|V|^{2} \mathrm{~d} \boldsymbol{x}, \mathrm{d} t \leq & \frac{1}{2} \int_{0}^{T} \liminf _{n \rightarrow \infty} \int_{\Omega}\left|D C_{n}(v)-z_{d}\right|^{2} \mathrm{~d} \boldsymbol{x} \mathrm{d} t \\
& +\frac{N}{2} \int_{0}^{T} \liminf _{n \rightarrow \infty} \int_{\Omega}\left|V_{n}\right|^{2} \mathrm{~d} \boldsymbol{x} \mathrm{d} t
\end{aligned}
$$

$J(V) \leq \liminf _{n \rightarrow \infty} J\left(V_{n}\right)$.

So that $J$ is inferior semi-continuous.

To proof the strong convexity, we choose $W$ and $V \in L^{2}(0, T ; H)$, then:

$$
\begin{aligned}
J\left(\frac{W+V}{2}\right)= & \frac{N}{2}\left\|\frac{W+V}{2}\right\|_{L^{2}(0, T ; H)}^{2} \\
& +\frac{1}{2} \int_{0}^{T} \int_{\Omega}\left|\frac{1}{2}\left(D C(V)-z_{d}\right)+\frac{1}{2}\left(D C(W)-z_{d}\right)\right|^{2} \mathrm{~d} \boldsymbol{x} \mathrm{d} t .
\end{aligned}
$$

Applying the first unequally Clarkson to the first term in right member, we obtain:

$$
\begin{aligned}
J\left(\frac{W+V}{2}\right) \leq & \frac{N}{2}\left[\frac{1}{2}\|W\|_{L^{2}(0, T ; H)}^{2}+\frac{1}{2}\|V\|_{L^{2}(0, T ; H)}^{2}-\left\|\frac{W-V}{2}\right\|_{L^{2}(0, T ; H)}^{2}\right] \\
& +\frac{1}{2} \int_{0}^{T} \int_{\Omega}\left|\frac{1}{2}\left(D C(v)-z_{d}\right)+\frac{1}{2}\left(D C(w)-z_{d}\right)\right|^{2} \mathrm{~d} \boldsymbol{x} \mathrm{d} t
\end{aligned}
$$


By applying the equality of the parallelogram to the second term the right member, we obtain:

$$
\begin{aligned}
J\left(\frac{W+V}{2}\right) \leq & \frac{N}{4}\|W\|_{L^{2}(0, T ; H)}^{2}+\frac{N}{4}\|V\|_{L^{2}(0, T ; H)}^{2}-\frac{N}{8}\|W-V\|_{L^{2}(0, T ; H)}^{2} \\
& +\frac{1}{4} \int_{0}^{T} \int_{\Omega}\left|D C(W)-z_{d}\right|^{2} \mathrm{~d} \boldsymbol{x} \mathrm{d} t+\frac{1}{4} \int_{0}^{T} \int_{\Omega}\left|D C(V)-z_{d}\right|^{2} \mathrm{~d} \boldsymbol{x} \mathrm{d} t \\
& -\frac{N}{8}\|D C(W)-D C(V)\|_{L^{2}(0, T ; H)}^{2} \cdot \\
J\left(\frac{W+V}{2}\right) \leq & \frac{N}{4}\|W\|_{L^{2}(0, T ; H)}^{2}+\frac{N}{4}\|V\|_{L^{2}(0, T ; H)}^{2}-\frac{N}{8}\|W-V\|_{L^{2}(0, T ; H)}^{2} \\
& +\frac{1}{4} \int_{0}^{T} \int_{\Omega}\left|D C(W)-z_{d}\right|^{2} \mathrm{~d} \boldsymbol{x} \mathrm{d} t+\frac{1}{4} \int_{0}^{T} \int_{\Omega}\left|D C(V)-z_{d}\right|^{2} \mathrm{~d} \boldsymbol{x} \mathrm{d} t \\
J\left(\frac{W+V}{2}\right) \leq & \frac{J(W)+J(V)}{2}-\frac{N}{8}\|W-V\|_{L^{2}(0, T ; H)}^{2} .
\end{aligned}
$$

And the differentiability of $J$ is proved by calculating the following limit:

$$
\lim _{\theta \rightarrow 0} \frac{J(V+\theta W)-J(V)}{\theta} .
$$

Let us denoted by $\breve{C}$ the trajectory corresponding to $V(t)+\theta W(t)$ then $\breve{C}$ satisfies the following equation:

$$
\begin{aligned}
& \left\{\tilde{C}(t)+\tilde{U} \cdot \nabla \tilde{C}(t)+d \Delta \tilde{C}(t)=G_{0}(t)+V(t)+\theta W(t) \quad \text { a.e } t \in[0, T]\right. \\
& \left\{\tilde{C}(0)=\tilde{C}_{0} \quad \text { in } \quad H\right. \text {. } \\
& J(V+\theta W)-J(V)=\frac{1}{2} \int_{0}^{T} \int_{\Omega}\left|D \tilde{C}-z_{d}\right|^{2} \mathrm{~d} \boldsymbol{x} \mathrm{d} t+\frac{N}{2} \int_{0}^{T}|V+\theta W|^{2} \mathrm{~d} \boldsymbol{x} \mathrm{d} t \\
& -\frac{1}{2} \int_{0}^{T} \int_{\Omega}\left|D C-z_{d}\right|^{2} \mathrm{~d} \boldsymbol{x} \mathrm{d} t-\frac{N}{2} \int_{0}^{T}|V|^{2} \mathrm{~d} \boldsymbol{x} \mathrm{d} t \\
& J(V+\theta W)-J(V) \\
& =\frac{1}{2} \int_{0}^{T} \int_{\Omega}\left[\left|D \tilde{C}-z_{d}\right|^{2}-\left|D C-z_{d}\right|^{2}\right] \mathrm{d} \boldsymbol{x} \mathrm{d} t+\frac{N}{2} \int_{0}^{T}\left[|V+\theta W|^{2}-|V|^{2}\right] \mathrm{d} \boldsymbol{x} \mathrm{d} t \\
& =\frac{1}{2} \int_{0}^{T}\left[\left(D \tilde{C}-z_{d}, D \tilde{C}-D C+D C-z_{d}\right)-\left(D C-z_{d}, D C-z_{d}\right)\right] \mathrm{d} t \\
& +\frac{N}{2} \int_{0}^{T}[(V+\theta W, V+\theta W)-(V, V)] \mathrm{d} t \\
& =\frac{1}{2} \int_{0}^{T}\left[\left(D \tilde{C}-z_{d}, D(\tilde{C}-C)+D C-z_{d}\right)-\left(D C-z_{d}, D C-z_{d}\right)\right] \mathrm{d} t \\
& +\frac{N}{2} \int_{0}^{T}\left[2 \theta(V, W)+\theta^{2}(W, W)\right] \mathrm{d} t \\
& =\frac{1}{2} \int_{0}^{T}\left[\left(D \tilde{C}-z_{d}, D(\tilde{C}-C)\right)+\left(D(\tilde{C}-C), D C-z_{d}\right)\right] \mathrm{d} t \\
& +\frac{N}{2} \int_{0}^{T}\left[2 \theta(V, W)+\theta^{2}(W, W)\right] \mathrm{d} t
\end{aligned}
$$

Let us designed by

$$
\hat{C}=\lim _{\theta \rightarrow 0} \frac{\tilde{C}-C}{\theta},
$$


then

$$
\begin{aligned}
& \begin{aligned}
\lim _{\theta \rightarrow 0} \frac{J(V+\theta W)-J(V)}{\theta}= & \frac{1}{2} \int_{0}^{T}\left[\left(D \tilde{C}-z_{d}, D \hat{C}\right)+\left(D \hat{C}, D C-z_{d}\right)\right] \mathrm{d} t \\
& +N \int_{0}^{T}(V, W) \mathrm{d} t
\end{aligned} \\
& J^{\prime}(V) W=\frac{1}{2} \int_{0}^{T}\left[\left(D \tilde{C}-z_{d}, D \hat{C}\right)+\left(D \hat{C}, D C-z_{d}\right)\right] \mathrm{d} t+N \int_{0}^{T}(V, W) \mathrm{d} t
\end{aligned}
$$

Note that if $\theta \rightarrow 0$ then $\tilde{C} \rightarrow C$

$$
J^{\prime}(V) W=\int_{0}^{T} \int_{\Omega}\left(D C-z_{d}\right) D \hat{C} \mathrm{~d} \boldsymbol{x} \mathrm{d} t+N \int_{0}^{T} \int_{\Omega} V W \mathrm{~d} \boldsymbol{x} \mathrm{d} t
$$

According to these properties of $J$ we conclude that problem (3) admits an unique minimum $W$ on $U_{a d}$.

From relation (47) we have the inequality:

$$
\|W-V\|_{L^{2}(0, T ; H)} \leq \frac{4}{N}[J(V)-J(W)] .
$$

As $U_{a d}=L^{2}(0, T ; H)$ then

$$
J^{\prime}(V) W=\int_{0}^{T} \int_{\Omega}\left(C-z_{d}\right) \hat{C} \mathrm{~d} x \mathrm{~d} t+N \int_{0}^{T} \int_{\Omega} V W \mathrm{~d} x \mathrm{~d} t
$$

search $\hat{C}$, i.e. the tangent model: In fact $\frac{(49)-(11)}{\theta}$, we obtain:

$$
\frac{\tilde{C}-\dot{C}}{\theta}+\frac{\tilde{U} \cdot \nabla \tilde{C}-U \cdot \nabla C}{\theta}-d \Delta \frac{\tilde{C}-C}{\theta}=w
$$

by tender $\theta \rightarrow 0$, we obtain

$$
\left\{\begin{array}{l}
\hat{C}+U \cdot \nabla \hat{C}-d \Delta \hat{C}=W \\
\hat{C}(\cdot, 0))=0
\end{array}\right.
$$

since $\tilde{U} \rightarrow U$ when $\theta \rightarrow 0$.

\subsection{Main Result 3}

Theorem 6. If $D$ is a surjective operator of the space $L^{2}(0, T ; V)$ in $L^{2}(0, T ; V)$ or $D$ is the identity application of $L^{2}(0, T ; V)$ then the control $V$ is given by:

$$
V=-N^{-1} P
$$

where $P$ is the solution of the adjoint equation.

First Case: We assume that $D$ is an injective operator define from the space in $L^{2}(0, T ; V) \rightarrow L^{2}(0, T ; H)$ and $z_{d} \in L^{2}(0, T ; V)$, then the adjoint equation is given by:

$$
\left\{\begin{array}{l}
-\dot{P}-U \cdot \nabla P-d \Delta P=C-z_{d} \\
P(T)=0
\end{array}\right.
$$

Seconde Case: $D$ is the identity operator define from $L^{2}(0, T ; V) \rightarrow L^{2}(0, T ; V)$ when $z_{d} \in L^{2}(0, T ; V)$ then adjoint equation is given by: 


$$
\left\{\begin{array}{l}
-\dot{P}-U \cdot \nabla P-d \Delta P=C-z_{d} \\
P(T)=0
\end{array}\right.
$$

when $z_{d} \in L^{2}(0, T ; H)$ the adjoint equation becomes:

$$
\left\{\begin{array}{l}
-\dot{P}-U \cdot \nabla P-d \Delta P=\left(\Delta_{x}+I\right)\left(C-z_{d}\right) \\
P(T)=0
\end{array}\right.
$$

Proof. The derivative given by (51) is unusable since for each test $W$ we must solving the tangent model (53). The introduction of the adjoint state allows us to obtain a explicit expression of $J^{\prime}$ [11] [12].

We first perform the scalar product of the first equation of (53) with $\mathrm{P}$ and then integrate by parts we obtain:

$$
\begin{aligned}
& \int_{0}^{T}(\hat{C}(t), P) \mathrm{d} t=\hat{C}(T) P(T)-C(0) P(0)-\int_{0}^{T}(P(t), \hat{C}(t)) \mathrm{d} t \\
& \begin{aligned}
\int_{0}^{T}(U \cdot \nabla \hat{C}(t), P) \mathrm{d} t & =\int_{0}^{T} \int_{\Omega} \nabla(U \hat{C}) P \mathrm{~d} t=\int_{0}^{T} \int_{\Gamma} U \hat{C} P \mathrm{~d} t-\int_{0}^{T} \int_{\Omega} \nabla(U P) \hat{C} \mathrm{~d} \boldsymbol{x} \mathrm{d} t \\
& =-\int_{0}^{T}(U \cdot \nabla P, \hat{C}) \mathrm{d} \boldsymbol{x} \mathrm{d} t
\end{aligned} \\
& \begin{aligned}
\int_{0}^{T}(\Delta \hat{C}, P) \mathrm{d} t & =\int_{0}^{T} \int_{\Omega} \Delta \hat{C} P \mathrm{~d} \boldsymbol{x} \mathrm{d} t=\int_{0}^{T} \int_{\Gamma} \hat{C} P \mathrm{~d} \boldsymbol{x} \mathrm{d} t-\int_{0}^{T} \int_{\Omega} \nabla \hat{C} \nabla P \mathrm{~d} \boldsymbol{x} \mathrm{d} t \\
= & -\int_{0}^{T} \int_{\Gamma} \hat{C} P \mathrm{~d} \boldsymbol{x} \mathrm{d} t+\int_{0}^{T} \int_{\Omega} \Delta P \hat{C} \mathrm{~d} \boldsymbol{x} \mathrm{d} t .
\end{aligned}
\end{aligned}
$$

with additional conditions on $P$ given by:

$$
P(\boldsymbol{x}, t=T)=0, \quad P(\boldsymbol{x}, t)=0 \quad \boldsymbol{x} \in \Gamma
$$

we obtain:

$$
\int_{0}^{T} \int_{\Omega}(-\dot{P}-U \cdot \nabla P-d \Delta P) \hat{C} \mathrm{~d} x \mathrm{~d} t=\int_{0}^{T} \int_{\Omega} W P \mathrm{~d} x \mathrm{~d} t
$$

By identifying this result with the first term of $J^{\prime}(V) W$, one obtains the adjoint equation according to the nature of the operator $D$ given by (55)-(57)

To characterize the control we consider the two cases given by relations (56), and (57). Then if the adjoint equation is given by the proof is the same for both cases $D$. The adjoint equation is given by:

$$
\left\{\begin{array}{l}
-\dot{P}-U \cdot \nabla P-d \Delta P=\left(C-z_{d}\right) \\
P(T)=0
\end{array}\right.
$$

In this case relation (51) becomes:

$$
J^{\prime}(V) W=\int_{0}^{T} \int_{\Omega}\left(C-z_{d}\right) \hat{C} \mathrm{~d} t+N \int_{0}^{T} \int_{\Omega} V W \mathrm{~d} x \mathrm{~d} t
$$

We form the scalar product between the first equation of the adjoint equation with $\hat{C}$ and we integrate over $[0, T]$, we obtain:

$$
\begin{aligned}
& \int_{0}^{T}(\dot{P}(t), \hat{C}) \mathrm{d} t=C(T) P(T)-C(0) P(0)-\int_{0}^{T}(\hat{C}(t), P(t)) \\
& \begin{aligned}
\int_{0}^{T}(U \cdot \nabla P(t), \hat{C}) \mathrm{d} t & =\int_{0}^{T} \int_{\Omega} \nabla(U P) \hat{C} \mathrm{~d} t=\int_{0}^{T} \int_{\Gamma} U P \hat{C} \mathrm{~d} t-\int_{0}^{T} \int_{\Omega} \nabla(U \hat{C}) P \mathrm{~d} \boldsymbol{x} \mathrm{d} t \\
& =-\int_{0}^{T}(U \cdot \nabla \hat{C}, P) \mathrm{d} \boldsymbol{x} \mathrm{d} t
\end{aligned}
\end{aligned}
$$




$$
\begin{aligned}
\int_{0}^{T}(\Delta P, \hat{C}) \mathrm{d} t & =\int_{0}^{T} \int_{\Omega} \Delta P \hat{C} \mathrm{~d} x \mathrm{~d} t=\int_{0}^{T} \int_{\Gamma} P \hat{C} \mathrm{~d} x \mathrm{~d} t-\int_{0}^{T} \int_{\Omega} \nabla P \nabla \hat{C} \mathrm{~d} x \mathrm{~d} t \\
& =-\int_{0}^{T} \int_{\Gamma} P \hat{C} \mathrm{~d} x \mathrm{~d} t+\int_{0}^{T} \int_{\Omega} \Delta \hat{C} P \mathrm{~d} x \mathrm{~d} t .
\end{aligned}
$$

We have therefore:

$$
\begin{aligned}
\int_{0}^{T}(-\dot{P}-U \cdot \nabla P-d \Delta P, \hat{C}) \mathrm{d} t & =\int_{0}^{T}(P, \hat{C}(t)+U \cdot \nabla \hat{C}+\Delta \hat{C}) \mathrm{d} t \\
& =\int_{0}^{T}(P, W) \mathrm{d} t=\int_{0}^{T} \int_{\Omega} P W \mathrm{~d} \boldsymbol{x} \mathrm{d} t .
\end{aligned}
$$

So that thus one can write $J^{\prime}$ as follows (51) give:

$$
\begin{aligned}
& J^{\prime}(V) W=\int_{0}^{T} \int_{\Omega}(-\dot{P}-U \cdot \nabla P-d \Delta P) \hat{C} \mathrm{~d} x \mathrm{~d} t+N \int_{0}^{T} \int_{\Omega} V W \mathrm{~d} x \mathrm{~d} t \\
& J^{\prime}(V) W=\int_{0}^{T} \int_{\Omega} P W \mathrm{~d} x \mathrm{~d} t+N \int_{0}^{T} \int_{\Omega} V W \mathrm{~d} x \mathrm{~d} t \\
& J^{\prime}(V) W=\int_{0}^{T} \int_{\Omega}(P+N V) W \mathrm{~d} x \mathrm{~d} t=0
\end{aligned}
$$

His equation had to be satisfied whatever the disturbance on $W$, especially if $W \neq 0$. Necessarily, we have:

$$
P+N V=0 .
$$

\section{Conclusions}

The aim of this paper is a mathematical analysis of an optimal control problem of surface water pollution by using a triplet of evolution adapted to order of derivations. This technique allowed us to propose theorems not only of existence and uniqueness of the control but also of the solution of the equation that governs the constraints which is nonlinear. Then we solved the control problem directly and gave an optimal control characterization by using the adjoint equation.

It is essential to remember that this study only allowed us to characterize the optimal control without giving an analytical expression of it. In a future work we will proceed to a numerical approximation of this model in order to propose solution to the decision makers.

\section{References}

[1] Gelfand, I.M. and Ya, N. (1964) Vilenkin; Generalized Functions. Applications of Harmonic Analysis. Vol. 4, Academic Press, New York.

[2] Pyatnitsky, E.S. and Rapoport, L.B. (1996) Criteria of Asymptotic Stability of Differential Inclusions and Periodic Motions of Time-Varying Nonlinear Control Systems. IEEE Transactions on Circuits and Systems, 43, 219-229. https://doi.org/10.1109/81.486446

[3] Ciarlet, P.G. (1982) Introduction à l'analyse numérique matricielle et à l'optimisation. Masson, Paris.

[4] Culioli, J.C. (1994) Introduction à l'optimisation. Editions Ellipses, Paris.

[5] Faurre, P. (1988) Analyse numérique. Notes d'optimisation, Ecole Polytechnique. Ellipse, Edition Marketing, Paris.

[6] Cannone, M. (2000) Nombres de Reynolds, stabilité et Navier-Stokes. Vol. 52, Banach Center Publications, Warszawa. 
[7] Brezis, H. (1968) Equations et inéquations non linéaires dans les espaces vectoriels en dualité. Annales de I Institut Fourier (Grenoble), 18, 115-175.

[8] Bonnans, J.F. (1998) Second Order Analysis for Control Constrained Optimal Control Problems of Semilinear Elliptic Systems. Applied Mathematics \& Optimization, 38, 303-325. https://doi.org/10.1007/s002459900093

[9] Aubin, J.P. (1963) Un théorème de compacité. Comptes Rendus de l'Académie des Sciences, Paris, 256.

[10] Clérin, J.-M. (2009) Problèmes de contrôle optimal du type bilinéaire gouvernés par des équations aux dérivées partielles d'évolution. Thèse, LANLG, Université d'Avignon.

[11] Lions, J.L. (1968) Contrôle optimal de systèmes gouvernés par des équations aux dérivées partielles. Dunod, Paris.

[12] Lions, J.-L. (1969) Quelques méthodes de résolution des problèmes aux limites non linéaires. Gauthier-Villars, Paris.

Submit or recommend next manuscript to SCIRP and we will provide best service for you:

Accepting pre-submission inquiries through Email, Facebook, LinkedIn, Twitter, etc. A wide selection of journals (inclusive of 9 subjects, more than 200 journals)

Providing 24-hour high-quality service

User-friendly online submission system

Fair and swift peer-review system

Efficient typesetting and proofreading procedure

Display of the result of downloads and visits, as well as the number of cited articles

Maximum dissemination of your research work

Submit your manuscript at: http://papersubmission.scirp.org/

Or contact am@scirp.org 